\title{
A NOTE ON THE SIZE-RAMSEY NUMBER OF LONG SUBDIVISIONS OF GRAPHS *
}

\author{
JAir Donadelli ${ }^{1}$, Penny E. HAXell ${ }^{2}$ \\ AND YOSHIHARU KOHAYAKaWA ${ }^{3}$
}

\begin{abstract}
Let $T_{s} H$ be the graph obtained from a given graph $H$ by subdividing each edge $s$ times. Motivated by a problem raised by Igor Pak [Mixing time and long paths in graphs, in Proc. of the 13th annual ACM-SIAM Symposium on Discrete Algorithms (SODA 2002) 321-328], we prove that, for any graph $H$, there exist graphs $G$ with $O(s)$ edges that are Ramsey with respect to $T_{s} H$.
\end{abstract}

Mathematics Subject Classification. 05C55, 05 D40.

\section{INTRODUCTION}

In this paper we are concerned with a numerical problem in Graph Ramsey Theory. All graphs in this note are finite and simple. The order of a graph $G$, denoted $|G|$, is the number of vertices in $G$ and the size of $G$, denoted $e(G)$, is the number of edges in $G$. Given an integer $q>0$ and graphs $G$ and $H$ we write $G \rightarrow(H)_{q}$ if $G$ contains a monochromatic copy of $H$ in any $q$-colouring of the edges of $G$. That is, for any $\varphi: E(G) \rightarrow\{1,2, \ldots, q\}$, there is a copy $H^{\prime} \subset G$ of the graph $H$ in $G$ such that $\varphi$ is constant on $E\left(H^{\prime}\right)$.

\footnotetext{
Keywords and phrases. The Size-Ramsey number, Ramsey theory, expanders, Ramanujan graphs, explicit constructions.

* This research was supported by the Thematic/PRONEX project FOCOS (Proc. FAPESP 03/09925-5). The first author was partially supported by CNPq (141633/98-0). The second author was partially supported by NSERC. The third author was partially supported CNPq (664107/97-4, 300334/93-1).

1 Departamento de Informática, Universidade Federal do Paraná, Centro Politécnico Caixa Postal 19081, 81531-980 Curitiba PR, Brasil; e-mail: jair@inf.ufpr.br

2 Department of Combinatorics and Optimization, University of Waterloo, Waterloo, Ontario N2L 3G1, Canada; e-mail: pehaxell@math.uwaterloo.ca

3 Instituto de Matemática e Estatística, Universidade de São Paulo, Rua do Matão 1010, 05508-090 São Paulo SP, Brasil; e-mail: yoshi@ime.usp.br
}

(c) EDP Sciences 2005 
The size-Ramsey number $\hat{r}_{q}(H)$ of a graph $H$ is the smallest number of edges in a graph $G$ such that $G \rightarrow(H)_{q}$, that is,

$$
\hat{r}_{q}(H)=\min \left\{e(G): G \rightarrow(H)_{q}\right\}
$$

Denote by $r_{q}(H)$ the (ordinary) Ramsey number of the graph $H$, that is, $r_{q}(H)=$ $\min \left\{n \in \mathbb{N}: K^{n} \rightarrow(H)_{q}\right\}$, where $K^{n}$ denotes the complete graph on $n$ vertices. As usual, let $\Delta(H)$ be the maximal degree in $H$ and let $\tau(H)$ be the minimal number of vertices of $H$ representing all its edges. The second inequality in

$$
\frac{1}{2} \Delta(H) \tau(H) \leqslant \hat{r}_{q}(H) \leqslant\left(\begin{array}{c}
r_{q}(H) \\
2
\end{array}\right)
$$

is immediate from the definitions of $\hat{r}_{q}(H)$ and $r_{q}(H)$; the first inequality in (1.1), due to Beck [4], follows from the following argument. Fix a graph $G$ such that $G \rightarrow$ $(H)_{q}$. Colour an edge of $G$ blue if at least one of its endpoints has degree at least $\Delta(H)$, otherwise colour the edge with any colour different from blue. As $G \rightarrow$ $(H)_{q}$ we conclude that there must exist a blue copy of $H$ in $G$, and this implies that the number of edges in $G$ has to be at least $(1 / 2) \Delta(H) \tau(H)$.

The investigation of size-Ramsey numbers was proposed by Erdős, Faudree, Rousseau and Schelp [8] in 1978 when, for example, they studied the size-Ramsey number of star forests and raised some questions concerning $\hat{r}_{2}\left(P^{n}\right)$, where $P^{n}$ is the path on $n$ vertices. Beck [4], using the probabilistic method, proved the surprising fact that $\hat{r}_{q}\left(P^{n}\right) \leqslant c n$, where $c=c(q)$ is a positive constant, settling a one-hundred-dollar question of Erdős.

Explicit examples of linear sized graphs that are Ramsey for $P^{n}$ were given by Alon and Chung [1], that is, they showed how to construct explicitly graphs $G$ with $O(n)$ edges such that $G \rightarrow\left(P^{n}\right)_{q}$.

Erdős and Graham [9] proved that the Ramsey number $r_{q}(T)$ of a tree $T$ satisfies

$$
\left\lfloor\frac{1}{2}(q+1)\right\rfloor(\tau(T)-1)<r_{q}(T) \leqslant 2 q e(T)+1 .
$$

It follows from the upper bound in (1.2) that the size-Ramsey number $\hat{r}_{q}(T)$ of a tree $T$ is $O\left(q^{2}|T|^{2}\right)$. In view of Beck's lower bound given in (1.1), we see that there exist trees $T$ that have size-Ramsey number $\Omega\left(|T|^{2}\right)$. However, as proved by Friedman and Pippenger [11] (see also [13,18]), the size-Ramsey number of trees of bounded degree is linear in the size of the tree. (See [15-17] for more on tree embeddings.) It was proved in [14] that cycles also have linear size-Ramsey numbers.

Beck [5] asked whether $\hat{r}_{2}(H)$ is always linear in the size of $H$ for graphs $H$ of bounded degree, and this was settled in the negative by Rödl and Szemerédi [30], who proved that there are graphs of order $n$, maximum degree 3 , and size-Ramsey number $\Omega\left(n(\log n)^{1 / 60}\right)$. The authors in [30] conjecture that, for some $\varepsilon=\varepsilon(\Delta)>$ 0 , we have

$$
n^{1+\varepsilon} \leqslant \hat{r}(n, \Delta) \leqslant n^{2-\varepsilon},
$$


where $\hat{r}(n, \Delta)$ is the maximum of $\hat{r}_{2}(H)$ over all graphs $H$ on $n$ vertices and of maximum degree at most $\Delta$. The upper bound in (1.3) has been proved by Kohayakawa, Rödl and Szemerédi (see [20]). For further recent results on sizeRamsey numbers, see $[10,26,27,29]$.

\subsection{Statement of the MAIN RESUlt}

Given a graph $H$ and a function $s: E(H) \rightarrow \mathbb{N}$, let the $s$-subdivision $T_{s} H$ of $H$ be the graph obtained from $H$ by replacing each edge $e$ in $H$ with an independent path of length $s(e)+1$ joining the endpoints of $e$. Clearly, this is equivalent to subdividing $s(e)$ times every edge $e$ of $H$. Thus, if $s$ is constantly 0 , then $T_{s} H=H$. If $s$ is bounded from below and from above by $\ell$ and $u$, then we write $\ell \leqslant s \leqslant u$.

Pak [25] conjectures that the size-Ramsey number of subdivided graphs is linear if the edges are subdivided enough times. His conjecture can be read as follows.

Conjecture 1.1. There is an absolute constant $c$ for which the following holds. For all integers $q \geqslant 2$ and $d$, there is a constant $C_{q, d}$ such that if $H$ is a graph with $\Delta(H)=d$ and $s$ is the constant function $s(e)=k$ for all $e \in E(H)$, where $k>$ $c \log m$ and

$$
m=\left|T_{s} H\right|=|H|+k|E(H)|,
$$

then $\hat{r}_{q}\left(T_{s} H\right) \leqslant C_{q, d}\left|T_{s} H\right|$.

Pak came close to proving Conjecture 1.1: his best bound for $\hat{r}_{q}\left(T_{s} H\right)$ is off only by a factor polylogarithmic in $m=\left|T_{s} H\right|$ (see [24,25]). Pak's approach is based on mixing times of random walks on graphs.

In this note we shall prove that, if a graph $H$ is fixed and $n$ is sufficiently large, then there exist pseudo-random graphs $G$ on $n$ vertices and with $O(n)$ edges that are Ramsey for $T_{s} H$ as long as $c \log n \leqslant s \leqslant C n$ for certain constants $c$ and $C>0$ (see Th. 1.2 below). Before stating our result precisely, let us introduce some notation and a definition.

Given a graph $G=(V, E)$, for any pair of disjoint sets $U, W \subset V$, we denote the set of edges and the number of edges in the bipartite subgraph induced by $U$ and $W$ in $G$ by $E_{G}(U, W)$ and

$$
e_{G}(U, W)=\left|E_{G}(U, W)\right|=\mid\{\{a, b\} \in E: a \in U \text { and } b \in W\} \mid .
$$

For any given reals $0<p \leqslant 1$ and $A>0$, we put $d=p n$ and say that a graph $G$ on $n$ vertices is $(p, A)$-uniform if

$$
\left|e_{G}(U, W)\right|-p|U||W| \mid \leqslant A \sqrt{d|U||W|}
$$

holds for all disjoint sets $U, W \subset V(G)$ such that $1 \leqslant|U| \leqslant|W| \leqslant d|U|$. In this note we shall be concerned with $(p, A)$-uniform graphs $G$ with constant average degree, and therefore with linearly many edges, that is, with $O(|G|)$ edges.

The main result of this note is the following. 
Theorem 1.2. For every integer $q \geqslant 2$, positive real $A$, and graph $H$, there exist positive constants $n_{0}, C_{0}, C_{1}$, and $C_{2}$ for which the following holds. If a graph $G$ on $n \geqslant n_{0}$ vertices is $(p, A)$-uniform with $d=p n \geqslant C_{0}$, then $G \rightarrow\left(T_{s} H\right)_{q}$ for any function $s$ such that $C_{1} \log n \leqslant s \leqslant C_{2} n$.

The proof of Theorem 1.2 is based on a variant of Szemerédi's well known regularity lemma [31]. An immediate consequence of the result above and the fact that sparse $(p, A)$-uniform graphs are abundant (see Lem. 2.4) is the following.

Corollary 1.3. For any graph $H$ and integer $q \geqslant 2$, there are constants $C=$ $C(H, q)$ and $s_{0}=s_{0}(H, q)$ such that if $s$ is the constant function $s(e)=k$ for all $e \in E(H)$, where $k \geqslant s_{0}$, then $\hat{r}_{q}\left(T_{s} H\right) \leqslant C\left|T_{s} H\right|$.

This note is organised as follows. In Section 2, we introduce the main technical lemmas that we shall need. In Section 3, we give an informal description of the proof of Theorem 1.2. Our proof strategy will be based on a version of Szemerédi's regularity lemma for edge-coloured sparse graphs. Theorem 1.2 is proved in Section 4. In what follows, we often tacitly assume that $n$ is large enough for our inequalities to hold; log stands for the natural logarithm and lg denotes the logarithm to the base 2 .

\section{Auxiliary Results}

\subsection{PATHS In EXPANDING BIPARTITE GRAPHS}

For the next two results, let us fix a bipartite graph $B=(U, W ; E)$, with vertex classes $U$ and $W$ and edge set $E$, and let positive reals $b$ and $f$ be given. We say that $B$ is $(b, f)$-expanding when, for every set $X$ of vertices of $B$ with $X \subset U$ or $X \subset W$, if $|X| \leqslant b$ then $|\Gamma(X)| \geqslant f|X|$, where, as usual, $\Gamma(X)$ is the neighbourhood of $X$, that is, the set of all vertices adjacent to some $x \in X$.

In this note, we shall use the following result, which is a variant of a well known lemma due to Pósa [28] (for a proof, see [12]).

Lemma 2.1. Let $b \geq 1$ be an integer. If the bipartite graph $B$ is $(b, 2)$-expanding, then $B$ contains a path $P^{4 b}$ on $4 b$ vertices.

We define the $\ell$-fold neighbourhood $\Gamma^{(\ell)}(x)$ of the vertex $x \in V(B)=U \cup W$ by

$$
\Gamma^{(\ell)}(x)=\{y \in V(B) \text { : there exists an } x-y \text { path of length } \ell \text { in } B\} .
$$

Lemma 2.2. If the bipartite graph $B$ is $(b, 4)$-expanding for some real $b \geq 1$, then $\left|\Gamma^{(\ell)}(x)\right| \geqslant 2^{\lfloor\ell / 2\rfloor}$ for all $x \in V(B)$ and all $1 \leqslant \ell \leqslant 2 \lg b+1$.

Proof. Fix $x$ and $\ell$ as in the statement of our lemma; say $x \in U$. We define a sequence of disjoint sets $S_{0}, S_{1}, \ldots, S_{\ell}$ inductively, where the $S_{t}(0 \leq t \leq \ell)$ are such that $(i) S_{t} \subset U$ if $t$ is even and $S_{t} \subset W$ if $t$ is odd, (ii) $\left|S_{t}\right|=2^{\lfloor t / 2\rfloor}$, and (iii) every $z \in S_{t}$ is joined to $x$ by a length $t$ path $P_{z}$ such that $\left|P_{z} \cap S_{s}\right|=1$ for $0 \leq s \leq t$. 
First let $S_{0}=\{x\}$. Suppose that $1 \leq s \leq \ell$ and that $S_{t}(0 \leq t<s)$ have already been defined satisfying $(i),($ ii), and (iii) above. Note that since $s \leq \ell$ we have $\left|S_{s-1}\right| \leq 2^{\lfloor(\ell-1) / 2\rfloor} \leq b$, and so $\left|\Gamma\left(S_{s-1}\right)\right| \geq 4\left|S_{s-1}\right| \geq 2^{s / 2+1}$. To define $S_{s}$, we proceed as follows. If $s$ is even we note that $T_{s}=\bigcup\left\{S_{2 i}: 0 \leq 2 i<s\right\}$ has cardinality $2^{s / 2}-1$, and choose $S_{s} \subset \Gamma\left(S_{s-1}\right) \backslash T_{s}$ of cardinality $2^{s / 2}$. If $s$ is odd we choose $S_{s} \subset \Gamma\left(S_{s-1}\right) \backslash T_{s}$ of size $2^{(s-1) / 2}$, where $T_{s}=\bigcup\left\{S_{2 i+1}: 1 \leq 2 i+1<s\right\}$. This completes the definition of the $S_{t}(0 \leq t \leq \ell)$. Now note that $S_{\ell} \subset \Gamma^{(\ell)}(x)$, and hence the result follows.

\subsection{SZEMERÉDi'S REGULARITY LEMMA}

We now describe a version of Szemerédi's regularity lemma for sparse graphs. Let a graph $G=(V, E)$ and a real number $0<p \leqslant 1$ be given. We define the $p$-density of a pair of non-empty, disjoint sets $U, W \subset V$ in $G$ by

$$
d_{G, p}(U, W)=\frac{e_{G}(U, W)}{p|U||W|} .
$$

For any $0<\varepsilon \leqslant 1$, the pair $(U, W)$ is said to be $(\varepsilon, G ; p)$-regular, or $(\varepsilon ; p)$-regular or even just $\varepsilon$-regular for short, if, for all $U^{\prime} \subset U$ with $\left|U^{\prime}\right| \geqslant \varepsilon|U|$ and all $W^{\prime} \subset W$ with $\left|W^{\prime}\right| \geqslant \varepsilon|W|$, we have

$$
\left|d_{G, p}(U, W)-d_{G, p}\left(U^{\prime}, W^{\prime}\right)\right| \leqslant \varepsilon .
$$

We say that a partition $\Pi=\left(V_{0}, V_{1}, \ldots, V_{k}\right)$ of $V$ is $(\varepsilon, k, G ; p)$-regular if $\left|V_{0}\right| \leqslant \varepsilon|V|$ and $\left|V_{i}\right|=\left|V_{j}\right|$ for all $i, j \in\{1,2, \ldots, k\}$, and, furthermore, at least $(1-\varepsilon)\left(\begin{array}{c}k \\ 2\end{array}\right)$ pairs $\left(V_{i}, V_{j}\right)$ with $1 \leqslant i<j \leqslant k$ are $(\varepsilon, G ; p)$-regular.

For technical reasons we introduce the following definition: we say that a graph $G$ on $n$ vertices is $(p, A)$-upper-uniform if, for $d=p n$, we have

$$
e_{G}(U, W) \leqslant p|U||W|+A \sqrt{d|U||W|}
$$

for all disjoint sets $U, W \subset V(G)$ such that $1 \leqslant|U| \leqslant|W| \leqslant d|U|$. In this note, we shall use the following result, which is a variant of Szemerédi's regularity lemma (see, e.g., $[19,21])$.

Lemma 2.3. For all real numbers $\varepsilon>0$ and $A \geqslant 1$ and all integers $k_{0}$, $q \geqslant 1$, there exist constants $n_{0}=n_{0}\left(\varepsilon, A, k_{0}, q\right)>0, d_{0}=d_{0}\left(\varepsilon, A, k_{0}, q\right)>0$, and $K_{0}=K_{0}\left(\varepsilon, A, k_{0}, q\right) \geqslant k_{0}$ such that the following holds. For every choice of $(p, A)$-upper-uniform graphs $G_{1}, G_{2}, \ldots, G_{q}$ on the same set $V$ of $n \geqslant n_{0}$ vertices, where $d=p n \geqslant d_{0}$, there exists a partition $\Pi=\left(V_{0}, \ldots, V_{k}\right)$ of $V$ with $k_{0} \leqslant k \leqslant K_{0}$ that is $\left(\varepsilon, k, G_{a} ; p\right)$-regular for every $a=1,2, \ldots, q$.

Let us remark that Lemma 2.3 holds under weaker hypotheses on the graphs $G_{a}$, but for the purpose of this note the above will do.

Let us now observe that, in a $(p, A)$-uniform graph $G$, the number of edges induced by a set $U$ of vertices is under tight control. Fix $U \subset V(G)$, let $u=|U|$, 
and set $s=\lfloor u / 2\rfloor$ and $t=\lceil u / 2\rceil$. Considering all the partitions $(S, T)$ of $U$ with $|S|=s$ and $|T|=t$, we have

$$
2 e(G[U])\left(\begin{array}{l}
u-2 \\
s-1
\end{array}\right)=\sum_{(S, T)} e(S, T)
$$

where the sum runs over all partitions $(S, T)$ as described above. We now apply (1.4) to $(2.2)$, to conclude that

$$
\begin{aligned}
e(G[U]) & =\frac{1}{2}\left(\begin{array}{l}
u-2 \\
s-1
\end{array}\right)^{-1}\left(\begin{array}{l}
u \\
s
\end{array}\right)\left\{p s t+O_{1}(A \sqrt{d s t})\right\} \\
& =p\left(\begin{array}{l}
u \\
2
\end{array}\right)+O_{1}\left(\frac{u(u-1)}{2 s t} A \sqrt{d}\right) \\
& =p\left(\begin{array}{l}
u \\
2
\end{array}\right)+O_{1}(A \sqrt{d} u)
\end{aligned}
$$

where, above, we write $O_{1}(x)$ for a term $y$ such that $|y| \leqslant x$. Therefore, for any $U \subset V(G)$, we have

$$
\left|e(G[U])-p\left(\begin{array}{c}
|U| \\
2
\end{array}\right)\right| \leqslant A \sqrt{d}|U| .
$$

We now observe that $(p, A)$-uniform graphs are abundant, as long as $A$ is a large enough constant. The following is proved in [14].

Lemma 2.4. For every $0<p=p(n) \leqslant 1$ the standard binomial random graph $G_{n, p}$ is $\left(p, \mathrm{e}^{2} \sqrt{6}\right)$-uniform with probability $1-o(1)$.

\subsection{LONG PATHS IN REGULAR 6-PARTITE GRAPHS}

Let us suppose $\rho_{0}, \alpha$, and $A$ are given positive real numbers. Fix a positive $\varepsilon$ such that

Let

$$
\varepsilon<\min \left\{\frac{1}{6}, \frac{\rho_{0}}{2\left(5+6 \rho_{0}\right)}\right\} .
$$

and choose $d$ satisfying

$$
\delta=\frac{\varepsilon}{\rho_{0}}+6 \varepsilon
$$

$$
d\left(\frac{\alpha \rho_{0} \delta}{A}\right)^{2} \geqslant 4
$$

Let $G$ be a $(p, A)$-upper-uniform graph on $n$ vertices where $p=d / n$. Let

$$
\mathbf{P}^{(m)}=\left(V_{1}, V_{2}, \ldots, V_{6}\right)
$$


be a 6 -tuple of pairwise disjoint sets $V_{i} \subset V(G)(1 \leqslant i \leqslant 6)$ such that

(i) $\left|V_{i}\right|=m \geqslant \alpha n$ for all $1 \leqslant i \leqslant 6$;

(ii) for all distinct $1 \leqslant i, j \leqslant 6$, the pair $\left(V_{i}, V_{j}\right)$ is $(\varepsilon, G ; p)$-regular of $p$-density $d_{G, p}\left(V_{i}, V_{j}\right)=d_{i, j} \geqslant \rho_{0}$.

From now on, for the results in this section, we suppose that $\mathbf{P}^{(m)}$ as above is fixed. Our next lemma is very similar to Lemma 3.2 in [14]; we include the proof for completeness.

Proposition 2.5. There exist $U_{i} \subset V_{i}(1 \leqslant i \leqslant 6)$ with $\left|U_{i}\right| \geqslant(1-5 \varepsilon) m$ for all $i$ such that

$$
\left|\Gamma(x) \cap U_{j}\right| \geqslant(1-\delta) d_{i, j} p m
$$

for any $x \in U_{i}$ and any $j \neq i$ with $1 \leqslant j \leqslant 6$.

Proof. Let us define a sequence

$$
\mathbf{P}^{(m)}(t)=\left(V_{1}(t), \ldots, V_{6}(t)\right) \quad(t=0,1,2, \ldots)
$$

in the following way. Start with $\mathbf{P}^{(m)}(0)=\left(V_{1}, \ldots, V_{6}\right)$. Suppose now that $t \geqslant 0$ and that we have defined $\mathbf{P}^{(m)}(t)$. If $(2.7)$ is satisfied for $U_{j}=V_{j}(t)(1 \leqslant j \leqslant 6)$, then we are home. Otherwise, take

$$
V_{i}(t+1)=V_{i}(t) \backslash\{x\}
$$

for some fixed $x \in V_{i}(t)$ and $i$ such that $\left|\Gamma(x) \cap V_{j}(t)\right|<(1-\delta) d_{i, j} p m$ for some $j \neq i$ with $1 \leqslant j \leqslant 6$; moreover, take $V_{k}(t+1)=V_{k}(t)$ for every $k \neq i$.

Let us suppose for a contradiction that, at some moment $T$, we have, without loss of generality, $\left|V_{1}(T)\right|<(1-5 \varepsilon) m$ and $\left|V_{j}(T)\right| \geqslant(1-5 \varepsilon) m$ for every $j \neq 1$.

As $\left|V_{1} \backslash V_{1}(T)\right|>5 \varepsilon m$ there exists $X \subset V_{1} \backslash V_{1}(T)$ with cardinality $>\varepsilon m$ such that, for some fixed $1<j \leqslant 6$, we have $\left|\Gamma(x) \cap V_{j}(T)\right|<(1-\delta) d_{1, j} p m$ for all $x \in X$. We conclude that

$$
e\left(X, V_{j}(T)\right)<(1-\delta) d_{1, j} p m|X|,
$$

which implies that the $p$-density of the pair $\left(X, V_{j}(T)\right)$ is

$$
d_{G, p}\left(X, V_{j}(T)\right)<\left(1-\frac{\varepsilon}{\rho_{0}(1-5 \varepsilon)}\right) d_{1, j}<d_{1, j}-\varepsilon,
$$

contradicting the regularity of the pair $\left(V_{1}, V_{j}\right)$.

The next result says that the $U_{i}$ 's in Proposition 2.5 induce expanding bipartite graphs.

Proposition 2.6. For every $i \neq j(1 \leqslant i, j \leqslant 6)$, the bipartite graph induced by $U_{i}$ and $U_{j}$ given in Proposition 2.5 is $\left((1-2 \delta) d_{i, j} m / f, f\right)$-expanding for any $0<f \leqslant\left(\delta \rho_{0} \alpha / A\right)^{2} d$. 
Proof. The proof will be by contradiction. Let $X \subset U_{i}$ be such that $|X| \leqslant$ $(1-2 \delta) d_{i, j} m / f$. Let $Y=\Gamma(X) \cap U_{j} \subset U_{j}$, with $j \neq i$, and suppose $|Y|<f|X|$.

By the upper-uniformity condition on $G$, we have

$$
e(X, Y) \leqslant p|X||Y|+A \sqrt{d|X||Y|}<p|X|(1-2 \delta) d_{i, j} m+A \sqrt{d|X||Y|},
$$

and, from (2.7), we deduce that

$$
e(X, Y)=e\left(X, U_{j}\right) \geqslant(1-\delta) d_{i, j} p m|X|
$$

Combining (2.8) and (2.9), we have that $\left(\delta d_{i, j} p m|X|\right)^{2}<A^{2} d|X||Y|$. Therefore

$$
|Y|>\frac{\left(\delta d_{i, j} \alpha p m|X|\right)^{2}}{A^{2} d|X|} \geqslant\left(\frac{\delta d_{i, j} \alpha}{A}\right)^{2} d|X| \geqslant f|X| .
$$

As we supposed that $|Y|<f|X|$, we have a contradiction.

Recall that $\mathbf{P}^{(m)}=\left(V_{1}, \ldots, V_{6}\right)$ as in $(2.6)$ is fixed. We shall now prove that there exist long paths connecting a positive fraction of the vertices in $V_{1}$ to a positive fraction of the vertices in $V_{6}$. Let

$$
\ell=\left\lfloor 2 \lg \left(\frac{(1-2 \delta) \rho_{0} m}{4}\right)\right\rfloor+1 \quad \text { and } \quad b=\left\lfloor 2(1-2 \delta) \rho_{0} m\right\rfloor-5 .
$$

Lemma 2.7. Let $s \in \mathbb{N}$ be such that $2 \ell+2 \leqslant s \leqslant 2 \ell+b+2$. Then there exist $X \subset V_{1}$ and $Y \subset V_{6}$ with $|X|,|Y| \geqslant \varepsilon m$ such that any $x \in X$ and any $y \in Y$ are endpoints of a path of length $s$ whose vertices belong to $\bigcup_{i=1}^{6} V_{i}$.

Proof. Let $s$ be an integer with $2 \ell+2 \leqslant s \leqslant 2 \ell+b+2$. Let $U_{i} \subset V_{i}(1 \leqslant i \leqslant 6)$ be the sets given by Proposition 2.5 and observe that Proposition 2.6 tells us that, pairwise, these sets induce $\left((1-2 \delta) \rho_{0} m / f, f\right)$-expanding bipartite graphs for any $0<f \leqslant 4$.

We now apply Lemma 2.1 to the bipartite graph induced by $\left(U_{3}, U_{4}\right)$ to get a path $P=x_{0}, x_{1}, \ldots, x_{b}$, where $b$ is as in (2.10) above. Without loss of generality, we may assume that $x_{0} \in U_{3}$. Put $i=s-2 \ell-2$, observe that $0 \leqslant i \leqslant b$, and consider $x_{i} \in V(P)$. As $x_{i} \in U_{j}$ for some $j \in\{3,4\}$ and $x_{0} \in U_{3}$, the condition in $(2.7)$ applied to $\left(U_{j}, U_{5}\right)$ and $\left(U_{2}, U_{3}\right)$ tells us that we may choose a neighbour $v$ of $x_{i}$ in $U_{5}$ and a neighbour $u$ of $x_{0}$ in $U_{2}$.

At this point we have a path of length $i+2$ connecting $u \in U_{2}$ to $v \in U_{5}$. Let $X=\Gamma_{\left(U_{1}, U_{2}\right)}^{(\ell)}(u) \cap U_{1}$ and $Y=\Gamma_{\left(U_{5}, U_{6}\right)}^{(\ell)}(v) \cap U_{6}$, where the $\ell$-fold neighbourhoods are taken in the bipartite graphs induced by $\left(U_{i}, U_{i+1}\right)(i \in\{1,5\})$. By the expansion properties of $\left(U_{i}, U_{i+1}\right)(i \in\{1,5\})$ and Lemma 2.2 with $\ell$ as defined in (2.10) above, we have

$$
|X|,|Y| \geqslant 2^{\lfloor\ell / 2\rfloor}>\frac{1}{8}(1-2 \delta) \rho_{0} m .
$$


Finally, by the choice of $\varepsilon$ (see (2.4)), we obtain that more than $(1-2 \delta) \rho_{0} m / 8 \geqslant$ $\varepsilon m$ vertices in $V_{1}$ are joined to more than $\varepsilon m$ vertices in $V_{6}$ by a path of length $s=2 \ell+i+2$, where $0 \leqslant i \leqslant b$, as required.

\subsection{EXTREMAL GRAPH THEORY - TURÁN'S THEOREM}

Below, in the proof of the main result, we shall use the following weaker form of the celebrated Turán Theorem (see, e.g., [7], Chap. 7, Ex. 8).

Theorem 2.8. For all integers $t$ and $k$, every graph $G$ on $k$ vertices and more than $\left(1-(t-1)^{-1}\right) k^{2} / 2$ edges contains a $K^{t}$ as a subgraph.

\section{Outline of the Proof of TheOrem 1.2}

Roughly speaking, the proof of Theorem 1.2 goes as follows. Let $H$ be a fixed graph and let $G$ be a $(p, A)$-uniform graph of order $n$, with $d=p n$ constant but large. Also, fix $s: E(H) \rightarrow \mathbb{N}$ in the range described in Theorem 1.2 and let $T_{s} H$ be an $s$-subdivision of $H$.

Suppose the edges of $G$ are partitioned into $q$ colour classes. We then have spanning subgraphs $G_{1}, \ldots, G_{q}$ of $G$, one for each colour, with $G=\bigcup_{1 \leq i \leq q} G_{i}$ and with each $G_{i}(p, A)$-upper-uniform. We apply to the $G_{i}(1 \leq i \leq q)$ the coloured version of Szemerédi's regularity lemma, Lemma 2.3 above, in order to obtain $t=6 e(H)+|H|$ subsets $V_{i}$ of $V(G)$ that are pairwise disjoint, of cardinality $m=\Omega(n)$ each, and with all the $\left(\begin{array}{l}t \\ 2\end{array}\right)$ pairs $\left(V_{i}, V_{j}\right) \varepsilon$-regular with $p$-density bounded away from 0 in some fixed colour $c \in\{1,2, \ldots, q\}$. (This may be accomplished applying the regularity lemma, Turán's theorem, and Ramsey's theorem in an appropriate fashion.)

Now, to each $x \in V(H)$, we associate one of the above sets $V_{i}$, which we denote by $V(x)$, with all the $V(x)(x \in V(H))$ distinct. We wish to find an embedding $\iota: V\left(T_{s} H\right) \rightarrow V\left(G_{c}\right)$ of $T_{s} H$ into $G_{c}$, the subgraph of $G$ spanned by the edges of colour $c$ (in what follows, the edges of the other colours are ignored). The vertices in $V(x)$ are the candidates for $\iota(x)$. The remaining $6 e(H)$ sets $V_{i}$ are divided into $e(H) 6$-tuples as in $(2.6)$, with one such 6 -tuple $\left(V_{1}^{(e)}, \ldots, V_{6}^{(e)}\right)$ for each edge $e \in E(H)$. For each $e$, the system $\left(V_{1}^{(e)}, \ldots, V_{6}^{(e)}\right)$ will "contain" a monochromatic path of colour $c$ of length $s(e)-1$ corresponding to $e$ in $T_{s} H$. Naturally, we shall have to connect this path of length $s(e)-1$ to $\iota(x) \in V(x)$ and $\iota(y) \in V(y)$, where $x$ and $y \in V(H)$ are the endpoints of $e \in E(H)$, to obtain the full $(s(e)+1)$-long path of $T_{s} H$ that corresponds to the edge $e$ of $H$.

To get the long paths in $\left(V_{1}^{(e)}, \ldots, V_{6}^{(e)}\right)$, we simply apply Lemma 2.7. Let us write $P_{e}$ for an $(s(e)-1)$-long path in $\left(V_{1}^{(e)}, \ldots, V_{6}^{(e)}\right)$ as given by that lemma.

To conclude the construction of the embedding of $T_{s} H$ into $G_{c}$, it suffices choose $\iota(x)$ for each $x \in V(H)$ in such a way that $\iota(x)$ is adjacent (in colour $c$ ) to the appropriate endpoints of the paths $P_{e}$ for all $e \in E(H)$ incident to $x$. As it turns out, if we fix the paths $P_{e}(e \in E(H))$ as described above, then it is not 


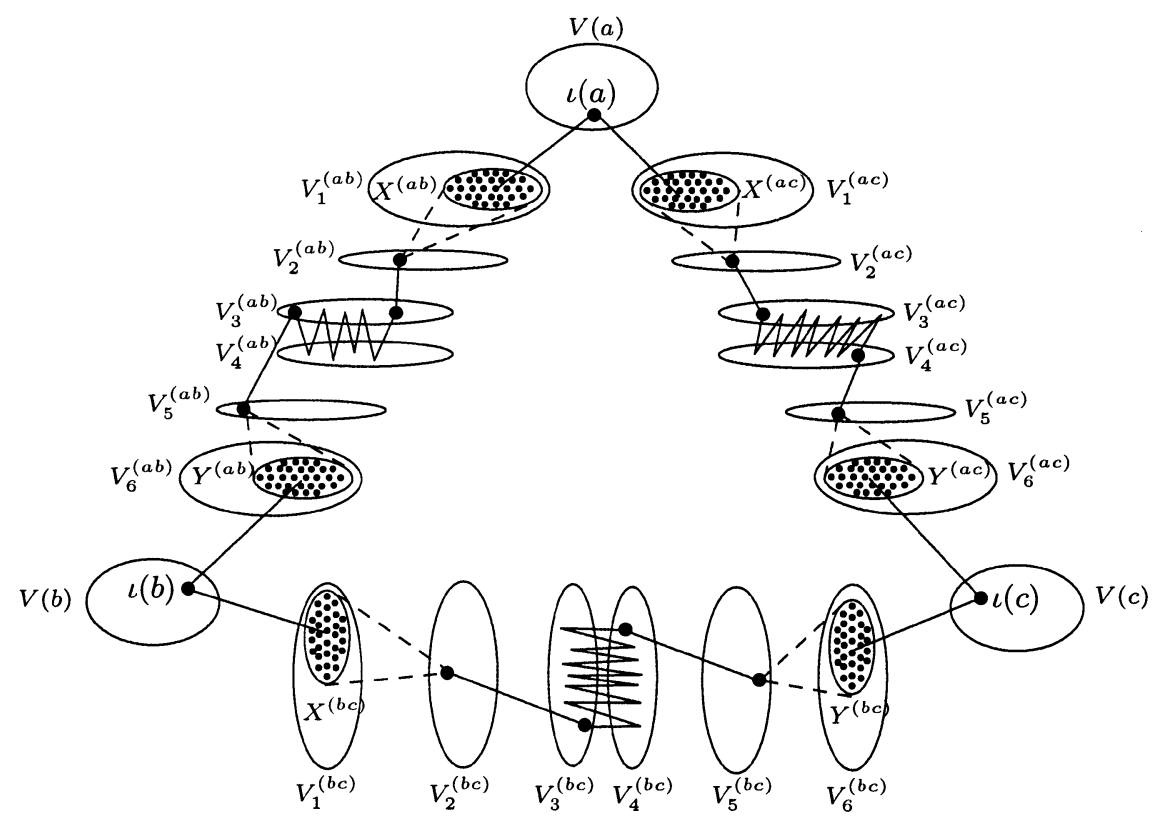

FIGURE 1. Schematic view of an embedding of a subdivision of the triangle with vertex set $\{a, b, c\}$.

clear whether this last task may be achieved. Therefore, our proof will follow a different route, although the outline above illustrates the approach quite closely.

Figure 1 illustrates the (very simple) case in which we wish to embed a subdivision of the complete graph on the vertex set $\{a, b, c\}$; that is, we wish to embed a cycle, which is seen as a subdivision of the triangle with vertices $a, b$, and $c$. The sets $V(a), V(b)$, and $V(c)$ are the sets of candidates for $\iota(a), \iota(b)$, and $\iota(c)$. The paths drawn with endpoints in the pairs $\left(V_{3}^{(e)}, V_{4}^{(e)}\right)(e \in\{a b, a c, b c\})$ correspond to the paths given by Lemma 2.1 and the dashed lines between $\left(V_{i}^{(e)}, V_{i+1}^{(e)}\right)$ $(e \in\{a b, a c, b c\}$ and $i \in\{1,5\})$ represent the iterated neighbourhood given by Lemma 2.2. The sets of endpoints of long paths given by Lemma 2.7 are represented by the filled regions in $V_{i}^{(e)}(e \in\{a b, a c, b c\}$ and $i \in\{1,6\})$.

\section{Proof of Theorem 1.2}

The core of the proof of Theorem 1.2 has two steps, and this section is organised accordingly (see Sects. 4.1 and 4.2 below). Before we come to the main part of the argument, we give the definitions of the constants that will be required. 
Let the constants $A \geqslant 1$ and $q \geqslant 2$ as in the statement of Theorem 1.2 be given. Also, fix a graph $H$ and write $\Delta$ for its maximum degree $\Delta(H)$. Let

$$
t=r_{q}\left(K^{|H|+6 e(H)}\right)
$$

be the ordinary Ramsey number for $K^{|H|+6 e(H)}$ with respect to $q$ colours. Moreover, put

Also, set

$$
\varrho=\frac{1}{2(t-1)} \quad \text { and } \quad \rho_{0}=\frac{\varrho}{q} \text {. }
$$

$$
\varepsilon=\min \left\{\frac{1}{20 q(t-1)}, \frac{\rho_{0}}{2\left(5+6 \rho_{0}\right)}, \frac{1}{\Delta+1}\right\} \quad \text { and } \quad k_{0}=\varepsilon^{-1}
$$

Let $d_{0}=d_{0}\left(\varepsilon, A, k_{0}, q\right)>0$ and $K_{0}=K_{0}\left(\varepsilon, A, k_{0}, q\right)$ be the constants given by Lemma 2.3. Let $\delta=\varepsilon / \rho_{0}+6 \varepsilon$ be as in (2.5) and put

$$
\alpha=\frac{1}{2 K_{0}}
$$

and

$$
\bar{d}_{0}=\max \left\{d_{0}, \frac{1}{\left(\rho_{0}-\varepsilon\right) \varepsilon \alpha}, 4\left(\frac{\alpha \rho_{0} \delta}{A}\right)^{-2}\right\} .
$$

We claim that we may choose

$$
C_{0}=\bar{d}_{0}, \quad C_{1}=6, \quad \text { and } \quad C_{2}=2(1-2 \delta) \alpha \rho_{0}
$$

in our theorem. This claim is verified in the remainder of the proof.

Let $0<p=p(n) \leqslant 1$ be such that $d=p n \geqslant C_{0}=\bar{d}_{0}$ and let us fix a $(p, A)$-uniform graph $G$ on $n$ vertices. Let $s: E(H) \rightarrow \mathbb{N}$ be an integer function satisfying $C_{1} \log n \leqslant s \leqslant C_{2} n$. We need to show that, as long as $n$ is large enough,

$$
G \rightarrow\left(T_{s} H\right)_{q}
$$

To prove (4.2), fix an arbitrary $q$-colouring of the edges of $G$. This colouring gives us a partition $E(G)=E_{1} \cup \cdots \cup E_{q}$ in a natural way. Our aim is to show that $G_{c}=G\left[E_{c}\right]$ contains $T_{s} H$ for some colour $1 \leqslant c \leqslant q$.

\subsection{The PRELIMINARY ARgument}

The argument in this section is standard, and is based on a combination of the regularity lemma, Turán's theorem, and Ramsey's theorem. We start by applying Lemma 2.3 with the constants $\varepsilon, A, k_{0}$, and $q$ as above to the family of $(p, A)$ upper-uniform graphs $G_{a}=G\left[E_{a}\right](a \in\{1,2, \ldots, q\})$. Let $\Pi=\left(V_{0}, V_{1}, \ldots, V_{k}\right)$ be an $\left(\varepsilon, k, G_{a} ; p\right)$-regular partition for all colours $a$, whose existence is guaranteed by 
that lemma. We call a pair $\left(V_{i}, V_{j}\right)(1 \leqslant i<j \leqslant k)$ regular and $G$-dense if it is $\left(\varepsilon, G_{a} ; p\right)$-regular for all $a \in\{1,2, \ldots, q\}$ and

$$
d_{G, p}\left(V_{i}, V_{j}\right) \geqslant \varrho .
$$

Let $m=\left|V_{1}\right|=\left|V_{2}\right|=\cdots=\left|V_{k}\right| \geqslant \alpha n$.

We now estimate the number of edges in $G$ that do not belong to pairs $\left(V_{i}, V_{j}\right)$ that are regular and $G$-dense using $(2.1)$ and (2.3). We need to take into account four classes of edges.

(i) The number of edges in $G$ that are incident to the vertices in $V_{0}$ is

$$
\begin{aligned}
\leqslant p\left(\begin{array}{c}
\varepsilon n \\
2
\end{array}\right)+A \sqrt{d} \varepsilon n+p \varepsilon(1-\varepsilon) n^{2} & +A \sqrt{d \varepsilon} n \\
& \leqslant \varepsilon p n^{2}+2 A \sqrt{d \varepsilon} n=2 \varepsilon\left(1+\frac{2 A}{\sqrt{d \varepsilon}}\right) \frac{n^{2} p}{2}
\end{aligned}
$$

(ii) The number of edges that belong to irregular pairs in some colour is

$$
\leqslant q \varepsilon\left(\begin{array}{l}
k \\
2
\end{array}\right)\left(p m^{2}+A \sqrt{d} m\right) \leqslant q \varepsilon\left(1+\frac{A k}{\sqrt{d}}\right) \frac{n^{2} p}{2} .
$$

(iii) The number of edges that belong to regular pairs that fail (4.3) is

$$
<\left(\begin{array}{l}
k \\
2
\end{array}\right) \varrho p m^{2} \leqslant \varrho \frac{n^{2} p}{2}
$$

(iv) The number of edges whose endpoints belong to the same $V_{i}(1 \leqslant i \leqslant k)$ is

$$
\leqslant k\left(p\left(\begin{array}{c}
m \\
2
\end{array}\right)+A \sqrt{d} m\right) \leqslant\left(\frac{1}{k}+\frac{2 A}{\sqrt{d}}\right) \frac{n^{2} p}{2} .
$$

Adding up the estimates in (4.4)-(4.7), we get that the number of edges in $G$ that do not belong to pairs $\left(V_{i}, V_{j}\right)$ that are regular and $G$-dense is

$$
\leqslant\left(3 \varepsilon+q \varepsilon+\frac{A k}{20 \sqrt{d}}+\varrho+\frac{1}{k}+\frac{2 A}{\sqrt{d}}\right) \frac{n^{2} p}{2} \leqslant\left((q+4) \varepsilon+\varrho+\frac{(k+40) A}{20 \sqrt{d}}\right) \frac{n^{2} p}{2} .
$$

Now, if at most $\left(1-(t-1)^{-1}\right)\left(k^{2} / 2\right)$ out of the $\left(\begin{array}{l}k \\ 2\end{array}\right)$ pairs $\left(V_{i}, V_{j}\right)(1 \leqslant i<j \leqslant k)$ are regular and $G$-dense, then

$$
<\left(1-\frac{1}{t-1}+\frac{A k}{\sqrt{d}}\right) \frac{n^{2} p}{2}
$$

edges of $E(G)$ are in regular and $G$-dense pairs, and $G$ has at most

$$
\left(1-\frac{1}{t-1}+(q+4) \varepsilon+\varrho+\frac{\left(21 K_{0}+40\right) A}{20 \sqrt{d}}\right) \frac{n^{2} p}{2} \leqslant\left(1-\frac{4 A}{\sqrt{d}}\right) \frac{n^{2} p}{2}
$$


edges. Inequality (2.3) tells us that $e(G) \geqslant p\left(\begin{array}{c}n \\ 2\end{array}\right)-A \sqrt{d} n$, which, combined with (4.8), yields a contradiction. Therefore, if we define a graph $R$ on the vertex set $\left\{V_{1}, V_{2}, \ldots, V_{k}\right\}$ whose edges are given by the pairs that are regular and $G$-dense, then

$$
e(R)>\left(1-\frac{1}{t-1}\right) \frac{k^{2}}{2} .
$$

By Theorem 2.8 (Turán's Theorem), it follows that $K^{t} \subset R$, that is, there exists a copy of the complete graph on $t$ vertices in $R$.

Clearly, for any $\left\{V_{i}, V_{j}\right\} \in E(R)$, there exists a colour $a \in\{1,2, \ldots, q\}$ such that $d_{G_{a}, p}\left(V_{i}, V_{j}\right) \geqslant \rho_{0}$. Colour the edges of this $K^{t} \subset R$ putting

$$
\operatorname{colour}\left(V_{i}, V_{j}\right)=\min \left\{a \in\{1,2, \ldots, q\}: d_{G_{a}, p}\left(V_{i}, V_{j}\right) \geqslant \rho_{0}\right\} .
$$

By Ramsey's theorem and the choice of $t$ (see (4.1)), there must exist a monochromatic copy of $K^{|H|+6 e(H)}$ in our $q$-coloured $K^{t} \subset R$. This corresponds to $|H|+$ $6 e(H)$ classes from $\left\{V_{1}, V_{2}, \ldots, V_{k}\right\}$ such that the edges of some colour, say $c \in$ $\{1,2, \ldots, q\}$, induce $\left({ }^{|H|+6 e(H)}\right) \varepsilon$-regular pairs of density at least $\rho_{0}$.

This finishes the first part of the proof. In what follows, we shall concentrate on the set $E_{c}$ of the edges of colour $c$ and on the $|H|+6 e(H)$ classes from $\left\{V_{1}, V_{2}, \ldots, V_{k}\right\}$ determined above.

\subsection{The Embedding of $T_{s} H$ IN $G_{c}=G\left[E_{c}\right]$}

We may suppose that

$$
K=\left\{V_{1}, V_{2}, \ldots, V_{|H|+6 e(H)}\right\}
$$

is our family of $|H|+6 e(H)$ vertex sets $V_{i}$ with all the $\left(\begin{array}{c}|H|+6 e(H) \\ 2\end{array}\right)$ pairs $\left(V_{i}, V_{j}\right)$ $\left(\varepsilon, G_{c} ; p\right)$-regular with density at least $\rho_{0}$. Recall that we are now ignoring all the edges that are not of colour $c$, that is, we restrict our attention to $G_{c}=G\left[E_{c}\right]$.

To each $x \in V(H)$, we associate a member $V_{i}$ of $K$, which we denote by $V(x)$, with all the $V(x)(x \in V(H))$ distinct. The remaining $6|E(H)|$ members in $K$ are split into $|E(H)|$ 6-tuples $\mathbf{P}_{e}^{(m)}=\left(V_{1}^{(e)}, \ldots, V_{6}^{(e)}\right)$ as in (2.6), one for each $e \in E(H)$.

Simple calculations show that $2 \ell+3 \leqslant C_{1} \log n \leqslant s \leqslant C_{2} n \leqslant 2 \ell+b+3$, where $\ell$ and $b$ are as in (2.10). Therefore

$$
2 \ell+2 \leqslant s(e)-1 \leqslant 2 \ell+b+2
$$

for all $e \in E(H)$. In view of (4.9), we may apply Lemma 2.7 to get, for each $e=x y \in E(H)$,

$$
\text { sets } Z(x, e) \subset V_{1}^{(e)} \text { and } Z(y, e) \subset V_{6}^{(e)}
$$


satisfying $|Z(x, e)|,|Z(y, e)| \geqslant \varepsilon m$ and so that any vertex in $Z(x, e)$ and any vertex in $Z(y, e)$ are the endpoints of a path of length $s(e)-1$ in the graph

$$
G_{c}\left[V_{1}^{(e)} \cup \cdots \cup V_{6}^{(e)}\right] .
$$

Before we proceed, we make two parenthetical remarks. In Figure 1, for convenience, we wrote $X^{(e)}$ and $Y^{(e)}$ for $Z(x, e)$ and $Z(y, e)$. In (4.10), we do not mind whether we match $x$ with $V_{1}^{(e)}$ and $y$ with $V_{6}^{(e)}$ or we match $x$ with $V_{6}^{(e)}$ and $y$ with $V_{1}^{(e)}$ (that is, we could also have $Z(x, e) \subset V_{6}^{(e)}$ and $Z(y, e) \subset V_{1}^{(e)}$ ).

To construct the embedding of $T_{s} H$ in $G_{c}=G\left[E_{c}\right]$, we make the following observations:

(*) If $(U, W)$ is an $(\varepsilon ; p)$-regular pair of density $\geqslant \rho_{0}$, then for any $Z \subset W$ with cardinality $|Z| \geqslant \varepsilon|W|$ we have $>(1-\varepsilon)|U|$ vertices $u \in U$ such that $|\Gamma(u) \cap Z| \geqslant\left(\rho_{0}-\varepsilon\right) p|Z|$.

(**) If $\left(U, W_{j}\right)(1 \leqslant j \leqslant \Delta)$ are $(\varepsilon ; p)$-regular pairs of density $\geqslant \rho_{0}$ and $Z_{j} \subset W_{j}$ $(1 \leqslant j \leqslant \Delta)$ are of cardinality at least $\varepsilon\left|W_{j}\right|$ each, then there exist at least $(1-\Delta \varepsilon)|U|$ vertices $u \in U$ such that $\Gamma(u) \cap Z_{j} \neq \emptyset$ for all $j \in\{1,2, \ldots, \Delta\}$.

The embedding $\iota: V\left(T_{s} H\right) \rightarrow V(G)$ of $T_{s} H$ into $G_{c}=G\left[E_{c}\right]$ that we seek is then easy to find. Below, we write $d(v)$ for the degree of $v \in V(H)$ in $H$ and write $\Gamma_{c}(w)$ for the neighbourhood of $w \in V\left(G_{c}\right)=V(G)$ in the graph $G_{c}$.

Let us first define $\iota(x)$ independently for all $x \in V(H)$ as follows: let $e_{1}=$ $x y_{1}, \ldots, e_{d(x)}=x y_{d(x)}$ be the edges incident to $x$ in $H$. Recall that we have the sets $Z\left(x, e_{1}\right), \ldots, Z\left(x, e_{d(x)}\right)$ associated to the edges $e_{i}(1 \leqslant i \leqslant d(x))$; see (4.10). We now apply $(* *)$ and take for $\iota(x)$ any vertex $x^{\prime}$ in $V(x)$ that has the property that $\Gamma_{c}\left(x^{\prime}\right) \cap Z\left(x, e_{i}\right) \neq \emptyset$ for all $1 \leqslant i \leqslant d(x)$. Moreover, fix a vertex $x_{i}^{\prime}$ in $\Gamma_{c}\left(x^{\prime}\right) \cap$ $Z\left(x, e_{i}\right)$ for all $1 \leqslant i \leqslant d(x)$. We have thus defined $\iota$ on $V(H) \cup \Gamma_{T_{s} H}(V(H)) \subset$ $V\left(T_{s} H\right)$, that is, on the vertices of $H$ and, for every $x \in V(H)$, on the leaves of the star of $d(x)$ rays centred at $x$.

To complete the definition of $\iota$, we need to 'join up' the leaves of the $|H|$ stars above by paths of the correct length, with one such path for each edge $e \in E(H)$. Fix $e=x y \in E(H)$. Recall that we have the 6 -tuple $\mathbf{P}_{e}^{(m)}=\left(V_{1}^{(e)}, \ldots, V_{6}^{(e)}\right)$ associated to $e$. Moreover, we have sets $Z(x, e) \subset V_{1}^{(e)}$ and $Z(y, e) \subset V_{6}^{(e)}$ such that any vertex in $Z(x, e)$ and any vertex in $Z(y, e)$ are the endpoints of a path of length $s(e)-1$ in the graph $G_{c}\left[V_{1}^{(e)} \cup \cdots \cup V_{6}^{(e)}\right]$. This implies that there is an appropriate $(s(e)-1)$-long path for each $e \in E(H)$, as required, and the definition of $\iota$ is complete.

\section{CONCLUding REMARKS}

Explicit examples of linear sized graphs that are Ramsey with respect to $T_{s} H$ are given by the Ramanujan graphs of Lubotzky, Phillips and Sarnak [22] and Margulis [23]. Let us briefly comment on the Lubotzky, Phillips, and Sarnak Ramanujan graphs $X^{p, q}$. Let $p$ and $q$ be unequal primes, both congruent 
to $1 \bmod 4$, with $p$ a square modulo $q$. The graph $X^{p, q}$ is $(p+1)$-regular of order $n=q\left(q^{2}-1\right) / 2$. The adjacency matrix of $X^{p, q}$ has second largest eigenvalue at most $2 \sqrt{p}$. From the results in [3] (Cor. 9.2.5) $|e(U, W)-((p+1) / n)| U\|W\| \leqslant$

$2 \sqrt{p|U||W|}$ for all disjoint subsets $U, W \subset V\left(X^{p, q}\right)$. Thus the $X^{p, q}$ are $((p+$ 1)/n,2)-uniform.

We close by mentioning two results concerning the ordinary Ramsey number: (i) a classical result of Chvátal, Rödl, Szemerédi, and Trotter [6] states that bounded degree graphs have linear Ramsey numbers; (ii) Alon [2] proved that, for any graph $H$, if $s: E(H) \rightarrow \mathbb{N}$ is such that $s(e) \geq 1$ for every $e \in E(H)$ (that is, every edge of $H$ is subdivided at least once), then the Ramsey number of $T_{s} H$ is $O\left(\left|T_{s} H\right|\right)$.

\section{REFERENCES}

[1] N. Alon and F.R.K. Chung, Explicit construction of linear sized tolerant networks. Discrete Math. 72 (1988) 15-19.

[2] N. Alon, Subdivided graphs have linear Ramsey numbers. J. Graph Theory 18 (1994) $343-347$.

[3] N. Alon and J.H. Spencer, The probabilistic method, 2nd edition, Ser. Discrete Math.Optim., Wiley-Interscience, John Wiley \& Sons, New York, 2000. (With an appendix on the life and work of Paul Erdös.)

[4] J. Beck, On size Ramsey number of paths, trees, and circuits. I. J. Graph Theory 7 (1983) $115-129$.

[5] J. Beck, On size Ramsey number of paths, trees and circuits. II. Mathematics of Ramsey theory, Springer, Berlin, Algorithms Combin. 5 (1990) 34-45.

[6] V. Chvátal, V. Rödl, E. Szemerédi and W.T. Trotter Jr., The Ramsey number of a graph with bounded maximum degree. J. Combin. Theory Ser. B 34 (1983) 239-243.

[7] R. Diestel, Graph theory. Springer-Verlag, New York (1997). Translated from the 1996 German original.

[8] P. Erdős, R.J. Faudree, C.C. Rousseau and R.H. Schelp, The size Ramsey number. Periodica Mathematica Hungarica 9 (1978) 145-161.

[9] P. Erdős and R.L. Graham, On partition theorems for finite graphs, Infinite and finite sets (Colloq., Keszthely, 1973; dedicated to P. Erdős on his 60th birthday), Vol. I. North-Holland, Amsterdam, Colloq. Math. Soc. János Bolyai 10 (1975) 515-527.

[10] R.J. Faudree and R.H. Schelp, A survey of results on the size Ramsey number, Paul Erdős and his mathematics, II (Budapest, 1999). Bolyai Soc. Math. Stud., János Bolyai Math. Soc., Budapest 11 (2002) 291-309.

[11] J. Friedman and N. Pippenger, Expanding graphs contain all small trees. Combinatorica 7 (1987) 71-76.

[12] P.E. Haxell, Partitioning complete bipartite graphs by monochromatic cycles. J. Combin. Theory Ser. B 69 (1997) 210-218.

[13] P.E. Haxell and Y. Kohayakawa, The size-Ramsey number of trees. Israel J. Math. 89 (1995) 261-274.

[14] P.E. Haxell, Y. Kohayakawa and T. Łuczak, The induced size-Ramsey number of cycles. Combin. Probab. Comput. 4 (1995) 217-239.

[15] P.E. Haxell and T. Łuczak, Embedding trees into graphs of large girth. Discrete Math. 216 (2000) 273-278.

[16] P.E. Haxell, T. Łuczak and P.W. Tingley, Ramsey numbers for trees of small maximum degree. Combinatorica 22 (2002) 287-320. Special issue: Paul Erdős and his mathematics. 
[17] T. Jiang, On a conjecture about trees in graphs with large girth. J. Combin. Theory Ser. B $\mathbf{8 3}$ (2001) 221-232.

[18] Xin Ke, The size Ramsey number of trees with bounded degree. Random Structures Algorithms 4 (1993) 85-97.

[19] Y. Kohayakawa, Szemerédi's regularity lemma for sparse graphs, Foundations of computational mathematics (Rio de Janeiro, 1997). Springer, Berlin (1997) 216-230.

[20] Y. Kohayakawa and V. Rödl, Regular pairs in sparse random graphs. I. Random Structures Algorithms 22 (2003) 359-434.

[21] Y. Kohayakawa and V. Rödl, Szemerédi's regularity lemma and quasi-randomness, in Recent advances in algorithms and combinatorics. CMS Books Math./Ouvrages Math. SMC, Springer, New York 11 (2003) 289-351.

[22] A. Lubotzky, R. Phillips and P. Sarnak, Ramanujan graphs. Combinatorica 8 (1988) 261-277.

[23] G.A. Margulis, Explicit group-theoretic constructions of combinatorial schemes and their applications in the construction of expanders and concentrators. Problemy Peredachi Informatsii 24 (1988) 51-60.

[24] I. Pak, Mixing time and long paths in graphs, manuscript available at http://www-math.mit.edu/ pak/research.html\#r (June 2001).

[25] I. Pak, Mixing time and long paths in graphs, in Proceedings of the 13th annual ACM-SIAM Symposium on Discrete Algorithms (SODA 2002) 321-328.

[26] O. Pikhurko, Asymptotic size Ramsey results for bipartite graphs. SIAM J. Discrete Math. 16 (2002) 99-113 (electronic).

[27] O. Pikhurko, Size Ramsey numbers of stars versus 4-chromatic graphs. J. Graph Theory 42 (2003) 220-233.

[28] L. Pósa, Hamiltonian circuits in random graphs. Discrete Math. 14 (1976) 359-364.

[29] D. Reimer, The Ramsey size number of dipaths. Discrete Math. 257 (2002) 173-175.

[30] V. Rödl and E. Szemerédi, On size Ramsey numbers of graphs with bounded degree. Combinatorica 20 (2000) 257-262.

[31] E. Szemerédi, Regular partitions of graphs, in Problèmes combinatoires et théorie des graphes (Colloq. Internat. CNRS, Univ. Orsay, Orsay, 1976). CNRS, Paris (1978) 399-401.

To access this journal online: www.edpsciences.org 